\title{
Erratum: Mouse and human strategies identify PTPN14 as a modifier of angiogenesis and hereditary haemorrhagic telangiectasia
}

\author{
Michael Benzinou, Frederic F. Clermont, Tom G.W. Letteboer, Jai-hyun Kim, Silvia Espejel, Kelly A. Harradine, \\ Juan Arbelaez, Minh Thu Luu, Ritu Roy, David Quigley, Mamie Nakayama Higgins, Musa Zaid, \\ Bradley E. Aouizerat, Johannes Kristian Ploos van Amstel, Sophie Giraud, Sophie Dupuis-Girod, Gaetan Lesca, \\ Henri Plauchu, Christopher C.W. Hughes, Cornelius J.J. Westermann \& Rosemary J. Akhurst
}

Nature Communications 3:616 doi: 10.1038/ncomms1633 (2012); Published 10 Jan 2012; Updated 23 Oct 2012.

This article contains four typographical errors in the abstract. Hereditary haemorrhagic telangiectasia is incorrectly abbreviated as HTT instead of HHT. The correct version of the abstract follows.

Hereditary haemorrhagic telangiectasia $(\mathrm{HHT})$ is a vascular dysplasia syndrome caused by mutations in transforming growth factor- $\beta$ / bone morphogenetic protein pathway genes, ENG and ACVRL1. HHT shows considerable variation in clinical manifestations, suggesting environmental and/or genetic modifier effects. Strain-specific penetrance of the vascular phenotypes of Eng $+/-$ and $T g f b 1^{-/}$mice provides further support for genetic modification of transforming growth factor- $\beta$ pathway deficits. We previously identified variant genomic loci, including $T g f b m 2$, which suppress prenatal vascular lethality of $T g f b 1^{-/-}$mice. Here we show that human polymorphic variants of PTPN14 within the orthologous TGFBM2 locus influence clinical severity of HHT, as assessed by development of pulmonary arteriovenous malformation. We also show that PTPN14, ACVRL1 and EFNB2, encoding EphrinB2, show interdependent expression in primary arterial endothelial cells in vitro. This suggests an involvement of PTPN14 in angiogenesis and/ or arteriovenous fate, acting via EphrinB2 and ACVRL1/activin receptor-like kinase 1. These findings contribute to a deeper understanding of the molecular pathology of HHT in particular and to angiogenesis in general. 